\title{
Malignancies in HIV
}

\author{
Nikolay A Belyakov*, Zinaida M Zagdyn², Vadim V Rassochin', Tatyana N Trofimova ${ }^{3}$, Alexey Y Kovelenov², \\ Robert Dubrow ${ }^{4}$, Robert Heimer ${ }^{4}$ \\ From $12^{\text {th }}$ International Conference on Malignancies in AIDS and Other Acquired Immunodeficiencies \\ (ICMAOI) \\ Bethesda, MD, USA. 26-27 April, 2010
}

We identified all known cases of malignancy among HIV-infected patients admitted to the AIDS Center hospital in Saint Petersburg in 2004-2009 via statistical cards completed for each discharged or dying case with a final clinical and/or autopsy diagnosis. From a total of 19,410 patients admitted during this period, we identified 38 cases $(0.2 \%)$. We randomly selected 42 patients without malignancy, also using statistical cards. The cancer cases included 15 (39.5\%) AIDS-defining malignancies (ADMs) (9 cases of non-Hodgkin's lymphoma [including one case of brain lymphoma], 3 cases of Kaposi's sarcoma, and 2 cases of invasive cervical cancer), and 23 (60.5\%) non-ADMs [Hodgkin's disease (7 cases), lung cancer (3 cases), breast, rectal, larynx, and brain cancer (2 cases each), oral, stomach and penis cancer (1 case each), and cancer of unknown primary site ( 2 cases)]. All cases were confirmed histologically; the two brain tumors were diagnosed as oligodendroglioma and ependymoma.

Most of the cancer patients were men (73.7\%), consistent with the population of patients visiting the AIDS Center. Most cases were diagnosed when patients sought medical care for cancer-related symptoms. About $60 \%$ of the subjects with malignancy died. Patients with ADMs were younger and had lower CD4 cell counts and lower HIV RNA loads than those with non-ADMs. There was no significant difference between the two groups in (a) the interval between HIV diagnosis and development of malignancy, (b) cancer survival, (c) HIV survival, and (d) frequency of death. The majority (55\%) of the patients had stage III-IV malignancies; over half of the ADM cases and about one-third of non-ADM cases were untreated. When comparing patients who had malignancy with those who had no cancer, the

\footnotetext{
*Correspondence: aidscenter@admiral.ru

${ }^{1}$ City AIDS Center, Saint Petersburg, Russia

Full list of author information is available at the end of the article
}

latter were younger, less likely drug users and smokers, and had higher CD4 cell counts and lower frequency of death.

\section{Conclusion}

The small number of malignancies we identified suggests that malignancies, especially ADMs, in HIVinfected patients are under-diagnosed in Saint Petersburg. The majority of cancers were diagnosed at their end stages, and almost half of cancers remained untreated. Patients with malignancy had more risk factors and a more severe clinical picture than those with no malignancy. It is imperative that we conduct further research on malignancies in HIV-infected persons in Saint Petersburg, with a focus on determining whether under-diagnosis is occurring and, if so, reasons for under-diagnosis. Programs to enhance cancer screening interventions in HIV-infected patients in Saint Petersburg may be needed.

\section{Acknowledgements \\ This article has been published as part of Infectious Agents and Cancer Volume 5 Supplement 1, 2010: Proceedings of the $12^{\text {th }}$ International Conference on Malignancies in AIDS and Other Acquired \\ Immunodeficiencies (ICMAOI). The full contents of the supplement are available online at http://www.biomedcentral.com/1750-9378/5?issue=S1}

\section{Author details}

${ }^{1}$ City AIDS Center, Saint Petersburg, Russia. ${ }^{2}$ AIDS Center, Leningrad Region, Russia. ${ }^{3}$ Mechnikov Medical Academy, Saint Petersburg, Russia. ${ }^{4}$ Yale School of Public Health, Yale School of Medicine, New Haven, CT, USA.

Published: 11 October 2010

doi:10.1186/1750-9378-5-S1-A52

Cite this article as: Belyakov et al:: Malignancies in HIV. Infectious Agents and Cancer 2010 5(Suppl 1):A52. 